\title{
Endocrine Health Problems Detected in 764 Patients Evaluated in a Late Effects Clinic
}

\author{
Maria Conceição Pereira Joana S. Pereira Daniela Dias Sara Donato \\ Duarte Salgado \\ Endocrine and Neurological Department, Portuguese Cancer Institute FG, Lisbon, Portugal
}

\section{Keywords}

Endocrine health problems · Survivor patients · Pediatric cancer · Late effects clinic

\begin{abstract}
Background: Many pediatric cancer survivors have endocrine conditions. After treatment with alkylating agents, steroids, methotrexate, and radiation, several endocrine dysfunctions may appear. Surveillance for late effects is recommended by guidelines worldwide. Objective: The objective of this study was to describe the endocrine outcomes of 764 patients followed during a 20 years' period in our out-patient clinic. Design: We retrospectively reviewed the medical records. Patients: The study included 764 patients whose oncological or hematological dangerous diseases appeared before they were 18 years old. Larger groups were constituted by leukemias, central nervous tumors, and lymphomas. Outcome Measures: The frequency and types of endocrine conditions were analyzed. Results: 1,091 endocrine conditions were observed in all groups. The most common types of endocrine conditions were problems with growth and the thyroid. We found puberty abnormalities and bone problems in third and fourth places of frequency. ACTH insufficiency was found in seventh place. Conclusion: Endocrine dysfunctions are very common in survivor populations. Endocrinologists should be aware of international guidelines and make an effort to optimize
\end{abstract}




\section{Case Reports in Oncology}

screening and treatment of endocrine effects of cancer therapy. The crucial period is the puberty with growth spurt failure and accelerated maturity both of which can bring future social and professional difficulties.

(C) 2017 The Author(s)

Published by S. Karger AG, Basel

\section{Introduction}

Improvements in therapy for pediatric cancers have resulted in increased survival. Overall, the 5-year survival rate for pediatric cancer patients now approaches $80 \%$ [1]. However, survivors are at risk of long-term complications of cancer therapy for the rest of their lives. Among adult survivors of pediatric cancer, $62.3 \%$ have been reported to have at least one health condition [2], and endocrine conditions are commonly reported. Various host and treatment factors that are associated with a higher risk of endocrine problems have been identified [3].

In March 2003, the Children's Oncology Group (COG) first published The Long-Term Follow-up Guidelines for Survivors of Childhood, Adolescents and Young Adult Cancers (COGLTFU Guidelines). These guidelines provide recommendations for screening and management of late effects of pediatric cancer therapy. The goal of guidelines is to promote health, standardize and enhance monitoring for late effects, and facilitate early detection and treatment. For example, screening for pituitary dysfunction is recommended after cranial irradiation or total body irradiation. Screening for thyroid dysfunction, nodules, and cancer is recommended after irradiation involving the head, neck, spine, or chest. Screening for gonadal dysfunction is recommended for patients exposed to radiation to the gonads, heavy metals, or alkylating chemotherapy. Screening for obesity and metabolic syndrome is recommended after cranial irradiation and total body irradiation. Screening for low body mineral density is recommended for survivors treated with hematopoietic stem cell transplant, methotrexate, and steroids. Problems with growth [4], weight [5], puberty and gonadal function [6], bone health [7], thyroid function [8], and adrenal function [9] have been described.

A Japanese study including 122 survivor patients with various cancer diagnoses identified an endocrine problem in $67 \%$ [10].

The objective of this study was to describe in detail the types of endocrine conditions detected in our endocrine clinic of survivor patients whose oncological disease had been diagnosed before they were 18 years old.

\section{Materials and Methods}

This study was a retrospective review of the clinical database from 764 patients (417 males and 347 females) evaluated at the Endocrine Rehabilitation Clinic (ERC) of the Portuguese Cancer Institute of Lisbon, Portugal, during the last 20 years. Patients are referred to the ERC by their oncologists and are offered an annual evaluation. Eligible patients attended at least 2 visits at the ERC. 


\section{Case Reports in Oncology}

The most common primary diagnoses were acute lymphoblastic leukemia (34.4\%), central nervous system tumors (27.61\%), lymphomas (19.63\%), aplasia/anemias (3.79\%), and renal/suprarenal tumors (3.79\%) (Table 1$)$.

Annual visits included physical examination and surveillance test according to the COGLTFU Guidelines. Endocrine function testing was obtained in some patients when clinically indicated. Data collected included cancer diagnosis, chemotherapeutic exposures, radiotherapy exposures, history of hematopoietic stem cell transplant, and surgery.

Endocrine health conditions of interest included problems of growth, gonadal function, adrenal function, thyroid health, weight, and bone health. For the purpose of this study, short stature was defined as a standing height less than the third percentile. Growth hormone (GH) deficiency was defined as a peak $\mathrm{GH}$ level $<7 \mathrm{ng} / \mathrm{mL}$ in response to insulin hypoglycemia test.

Patients attending the ERC with a history of at least 40 Gy of radiation to the brain, orbit, ear, nasopharynx, or waldeyer's ring were screened for adrenal insufficiency. Patients with abnormal cortisol levels $<10 \mu \mathrm{g} / \mathrm{dL}$ were evaluated with dynamic adrenal function testing with standard hypoglycemic test. Adrenal insufficiency was defined as a peak $<18 \mu \mathrm{g} / \mathrm{dL}$.

Precocious puberty was defined as puberty at an abnormally early age $(<8$ years for girls and 9 years for boys). Delayed puberty was defined as the absence of secondary sexual characteristics by the age of 15 years in males (specifically phallic development and pubic hair, not only testicular volume) and by the age of 13 years in females. Hypogonadism was defined as a clinically significant lack of the production of sex steroids requiring exogenous replacement of these hormones and was subclassified into hypergonadotropic or hypogonadotropic. Gonadal function was assessed in females at 13 years or older and in males at 14 years or older with prior exposure to alkylating chemotherapy or predisposing radiation. Additionally, gonadal function was assessed in those with delayed puberty, delayed menarche, or abnormal menses. Gonadal function was assessed by measurement of luteinizing hormone, follicle-stimulating hormone, estradiol (in females), and total and free testosterone (in males). Low levels were confirmed. Overweight was defined as a body mass index greater than the 95th percentile for age.

Bone mineral density was evaluated for 20-year-old or older survivors: a T score less than -1.5 was defined as low bone mineral density, and a $\mathrm{T}$ score less than -2.5 was defined as severely low bone mineral density.

\section{Results}

In this study we found several endocrine problems. They were found in pediatric cancer survivors after surgery (34.8\%), chemotherapy (99.47\%), radiotherapy (53.4\%), and bone marrow transplantation $(37.43 \%)$. The most common types of endocrine problems were growth problems $(35.99 \%)$, thyroid pathology $(28.4 \%)$, gonadal dysfunction $(26.96 \%)$, infertility or subfertility (17\%), and bone mass deficiency (9.81\%). Table 2 displays the frequency of various types of endocrine problems in affected survivors. Treatment with radiation, stem cell transplant, and older age were associated with a significantly higher hazard. 


\section{Case Reports in Oncology}

\section{Discussion}

Prior research in survivor patients has shown that in pediatric cancer survivors, the most common types of health conditions after pediatric cancer therapy are endocrine and neuropsychological [11]. This study demonstrated a large number and wide range of endocrine health conditions in pediatric cancer survivors detected in only 1 outpatient clinic.

Many of the outcomes in this study are not surprising, such as the association of radiation therapy and stem cell transplant with the occurrence of endocrine health conditions [11-13]. Patients who require stem cell transplantation due to relapse have a high cumulative exposure to chemotherapy and radiation. Depending on the dose and fields treated in an individual cancer survivor, the risk of endocrine disorders may be lower or higher.

Limitations of this study include the absence of statistical treatment data. However, it demonstrated the high prevalence of endocrine disorders in oncological patients. Our reported percentages of endocrine conditions may be higher than those in clinics in which an endocrinologist is not directly available for patient evaluation. In our cohort, survivors underwent targeted screening for endocrine problems based on their prior treatment exposures or if they exhibited signs and symptoms. Research is needed surrounding controversial aspects of endocrine care of cancer survivors, including screening for and diagnosis of adrenal insufficiency and assessment of fertility risk.

Short stature may cause deep and complex psychological problems which can have an impact on an individual's daily life, and Tanner grade I/II in adolescents may cause great mental suffering. Panhypopituitarism and diabetes insipidus have a significant, real, shortterm, life-threatening effect, and their diagnosis must be done in due time. Bone mass deficiency will be a burden for the survivors' future. Diabetes plus dyslipidemia will strongly contribute to vascular disease which is very frequent in this population with emergence of the vascular problems at young ages.

In summary, endocrine problems are among the most common health conditions affecting cancer survivors. It is essential that endocrinologists familiarize themselves with the COG-LTFU Guidelines and with the endocrine sequelae frequently seen in survivors of pediatric cancer.

\section{Statement of Ethics}

The study was conducted applying all principles of Ethics.

\section{Disclosure Statement}

The authors have nothing to declare. 


\section{Case Reports in Oncology}

Pereira et al.: Endocrine Health Problems Detected in 764 Patients Evaluated in a Late Effects Clinic

\section{References}

1 Linabery AM, Ross JA: Childhood and adolescent cancer survival in the US by race and ethnicity for the diagnostic period 1975-1999. Cancer 2008;113:2575-2596.

-2 Oeffinger KC, Mertens AC, Skylar CA, Kawashima T, Hudson MM, Meadows AT, Fiedman DL, Marina N, Hobbie W, Kadam-Lottick NS, Schwarts CL, Leisenring W, Robison LL: Chronic health conditions in adult survivors of childhood cancer. N Engl J Med 2006;355:1572-1582.

- Hudson MM, Mulrooney DA, Bowers DC, Skylar CA, Green DM, Donaldson SS, Oeffinger KC, Neglia JP, Meadows AT, Robinson LL: High-risk populations identified in Childhood Cancer Survivors Study investigations: implications for risk-based surveillance. J Clin Oncol 2009;27:2405-2414.

- 4 Noorda EM, Somers R, Van Leeuwen FE, Vulsma T, Behrendt H: Adult height and age at menarche in childhood cancer survivors. Eur J Cancer 2001;37:605-612.

5 Nathan PC, Jovcevska V, Ness KK, Mammone D'Agostino N, Staneland P, Urbach SL, Barron M, Barrera M, Greenberg ML: The prevalence and overweight and obesity in pediatric survivors of cancer. J Pediatr 2006;149:518-525.

6 Marquis A, Kuehni CE, Strippoli MP, Kuhne T, Brazolla: Sperm analysis of patients after successful treatment of childhood acute lymphoblastic leukemia with chemotherapy. Pediatr Blood Cancer 2010;55:208-210.

7 Thomas IH, Donohue JE, Ness KK, Dengel DR, Baker KS, Gurney JG: Bone mineral density in young adult survivors of acute lymphoblastic leukemia. Cancer 2008;113:3248-3256.

8 Chow EJ, Friedman DL, Stovall M, Yasui Y, Whitton JA, Robison LL, Sklar CA: Risk of thyroid dysfunction and subsequent thyroid cancer among survivors of acute lymphoblastic leukemia: a report from Childhood Cancer Survivor Study. Pediatr Blood Cancer 2009;53:432-437.

-9 Patterson BC, Truxillo L, Wasilewski-Masker K, Mertens AC, Meacham LR: Adrenal function testing in pediatric cancer survivors. Pediatr Boold Cancer 2009;53:1302-1307.

10 Miyoshi Y, Ohta H, Hashii Y, Tokimasa S, Namba N, Mushiake S, Hara J, Ozono K: Endocrinological analysis of 122 Japanese childhood cancer survivors in a single hospital. Endocr J 2008;55:1055-1063.

11 Wasilewski-Masker K, Mertens AC, Patterson B, Meacham LR: Severity of health conditions identified in a pediatric cancer survivor program. Pediatr Blood Cancer 2010;54:976-982.

12 Gurney JG, Kadan-Lottick NS, Packer RJ, Neglia JP, Sklar CA, Puniko JA, Stovall M, Yasui Y, Nicholson HS, Wolden S, McNeil DE, Mertens AC, Robinson LL: Endocrine and cardiovascular late effects among adult survivors of childhood brain tumors: Childhood Cancer Survivor Study. Cancer 2003;97:663-673.

-13 Rohrer TR, Beck JD, Grabenbauer GG, Fahlbusch R, Buchfelder M, Dorr HG: Late endocrine sequelae after radiotherapy of pediatric brain tumors are independent of tumor location. J Endocrinol Invest 2009;32:294-297. 


\section{Case Reports in Oncology}

Table 1. Number and types of oncological diseases in the total sample

\begin{tabular}{lcc}
\hline Oncological disease & Patients, $n$ & Percentage of sample \\
\hline Leukemia & 263 & $34.4 \%$ \\
Central nervous system tumor & 211 & $27.61 \%$ \\
Lymphoma & 150 & $19.63 \%$ \\
Anemia/aplasia & 29 & $3.79 \%$ \\
Renal/suprarenal tumor & 29 & $3.79 \%$ \\
Dangerous inflammatory disease & 20 & $2.61 \%$ \\
Ovary/pelvic tumor & 18 & $2.35 \%$ \\
Cavum carcinoma & 14 & $1.83 \%$ \\
Others & 33 & $4.3 \%$ \\
\hline
\end{tabular}

Table 2. Percentage of subjects affected by the more common types of endocrine problems overall

\begin{tabular}{lll}
\hline Endocrine dysfunction & Patients, $n$ & Percentage of sample \\
\hline Growth hormone disorder & 275 & $35.99 \%$ \\
Thyroid pathology & 217 & $28.4 \%$ \\
Pubertal dysfunction & 206 & $26.96 \%$ \\
Infertility/subfertility & 130 & $17 \%$ \\
Bone mass deficiency & 75 & $9.81 \%$ \\
Diabetes/dyslipidemia/obesity & 59 & $7.7 \%$ \\
Panhypopituitarism & 40 & $5.23 \%$ \\
Mammary dysfunction & 37 & $4.84 \%$ \\
Hyperparathyroidism & 17 & $2.22 \%$ \\
Diabetes insipidus & 35 & $4.6 \%$ \\
\hline
\end{tabular}

